\title{
Electromagnetic Powder Deposition Experiments
}

\author{
R.C. Zowarka, J.R. Uglum, J.L. Bacon, M.D. Driga, \\ R.L. Sledge, and D.G. Davis \\ Center for Electromechanics, The University of Texas at Austin
}

\begin{abstract}
The Department of Defense (DoD) and commercial entities are dependent on chemical plating and coating processes to replace worn or eroded material on damaged parts. Logistics Centers have been forced to consider replacement materials for repair operations due to the tightening of government regulations on the use of toxic and hazardous materials.

This paper describes a new process capable of fulfilling many of these requirements. Existing state of the art thermal spray processes (HVOF, D-gun, plasma spray) are limited to powder velocities of about $1 \mathrm{~km} / \mathrm{s}$ because they rely on the thermodynamic expansion of gases. A new thermal spray process using electromagnetic forces can accelerate powder particles to a final velocity in excess of $2 \mathrm{~km} / \mathrm{s}$. At this velocity, powder particles have sufficient kinetic energy to melt their own mass and an equivalent substrate mass on impact. The energetics of the process allow fusion bonding of greater strength than that created by low velocity processes as well as improved coating density.

This paper will describe the laboratory system designed and constructed to conduct proof of principle experiments. Results of the experiments will be presented along with high speed photographs of powder particles confirming system modeling and performance. The paper will conclude with a discussion of the future direction of the program.
\end{abstract}

\section{INTRODUCTION}

The Electromagnetic Powder Deposition (EPD) process was developed at The University of Texas at Austin Center for Electromechanics (UT-CEM) as a method of imparting high velocities to powder particles. Fig. 1 compares several thermal spray processes in terms of particle velocity and gas temperature and show how EPD is predicted to perform. The data for the other processes was obtained from a paper describing a "Cold Gas-Dynamic Method."[1] What is unique to the EPD approach is that the use of electromagnetic railgun force means that the gas flow velocity can be as high as desired and is not limited by any chemical or thermodynamic constraints. The railgun process is combined with a gas-dynamic mechanism called a snowplow [2] to produce controllable bursts of

Manuscript received May 1, 1998.

R. Zowarka may be contacted at 512-471-4496, fax 512-471-0781, r.zowarka@mail.utexas.edu.

This research was sponsored by the United States Air Force, Tinker Air Force Base at Oklahoma City, through a contract administered by ARINC Corporation. gas with the speed and duration required to accelerate finite segments of dispersed powder to the conditions required for plating purposes. The process has promise of greatly improving bond strength and coating density if empirical scaling trends continue with increasing velocity.

The railgun is filled with an ionizable gas, and a radio frequency (rf) excited cavity at the breech of the accelerator provides a line source of plasma. A high energy electrical pulse, provided by a pulsed energy source, expands the line source into a planar arc which is driven forward by electromagnetic forces. The arc is an efficient snowplow sweeping the gas in the bore to a final velocity approximately twice desired powder velocity. The gun length and current pulse is tailored such that the plasma arc is extinguished as the gas column reaches the powder ports into the railgun bore. The hot plasma does not interact with the powder or the substrate surface. The shocked gas passes over a powder cloud introduced near the end of the gun and accelerates the powder through drag forces. The electrical and powder discharge frequency can be

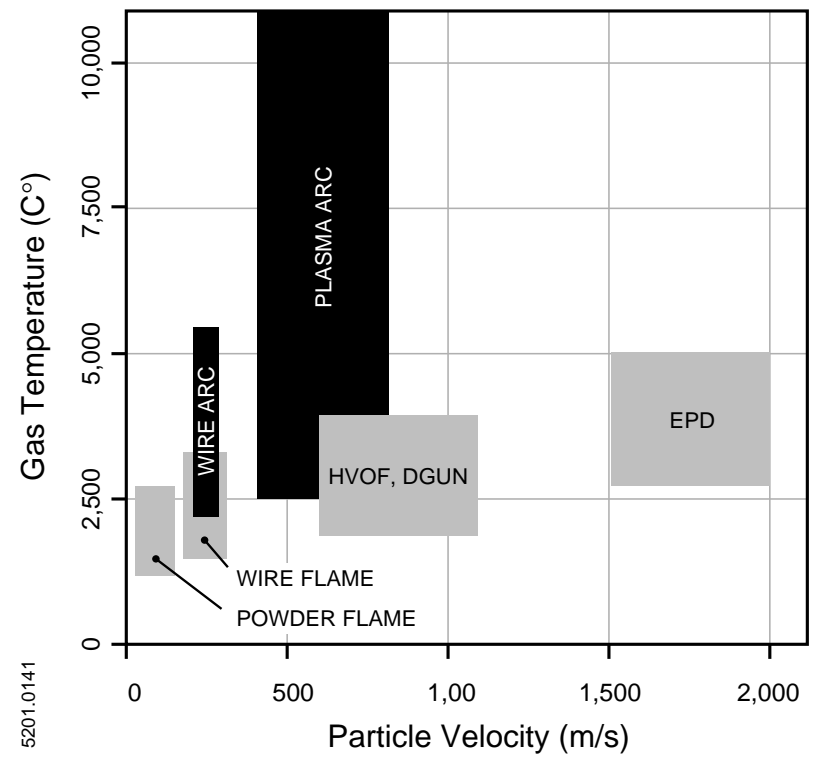

Fig. 1. A comparison of particle velocity and gas temperature for several thermal spray processes. 
adjusted so that the deposition rate and thermal input to the substrate can be controlled.

The new process uses a regenerating line source of plasma at the breech of the gun as opposed to a wire or a fuse to start the arc. This allows the process to be rep ratable and requires no wire feed or fuse loader. Powder in this process is introduced continuously at the muzzle of the gun at low velocity and only accelerated powder can reach the target.

The process has the ability to control the thermal input to the substrate because it is pulse-driven as opposed to continuous. The process of forming and accelerating the snowplow arc can be unstable. A special current pulse to drive the process has been specified to avoid plasma instabilities. The process has been designed to operate at atmospheric pressure for ease of use and cost savings. A special rf excitation source and arc generation cavity have been designed to allow the initiation plasma to form at atmospheric pressure. A special manifold has been designed for the end of the gun to keep the substrate flooded with inert gas thereby preventing target oxidation. Because the process uses a snowplowed gas column to accelerate the powder with drag forces, conducting and nonconducting powders may be sprayed.

This process can be used to build up material of parent material strength because it has the potential to create a fusion bond with the substrate. It can build up the material with less heat input than a welding process therefore mitigating substrate warpage. The more energetic impact will create denser coatings. It can be used to apply chrome to substrates therefore avoiding the generation of environmentally hazardous hexavalent chrome, a byproduct of electroplating. Due to the improved bond strength, material build-up may be possible, allowing the formation of macro structures.

\section{ACCELERATOR DESCRIPTION}

The EPD spayer, a railgun, consists of two metallic rails with insulating sidewalls separating them. The bore is filled with an ionizable gas, and an radio frequency (rf) excited cavity at the breech of the accelerator provides a line source of plasma.[3] A high energy electrical pulse, provided by a pulsed energy source, expands the line source into a planar arc which is driven forward by electromagnetic forces. Fig. 2 shows a simple railgun and the electrical currents and magnetic fields which interact to create the Lorentz force (the cross product of the current density vector and the magnetic field vector). The arc is an efficient snowplow sweeping the gas in the bore to a final velocity approximately twice the desired powder velocity. This shocked gas passes over a powder cloud introduced near the end of the gun and accelerates the powder through drag forces. The electrical and powder discharge frequency can be adjusted so that the deposition rate and thermal input to the substrate can be controlled.

The proposed technical program evolved from considerations of the snowplow mechanism driven by electromagnetic Lorentz railgun forces. It can be shown [4] that current requirements are related to gas velocity by the relation

$$
I=16 V_{\text {gas }} \sqrt{\frac{\rho_{\text {gas }} A}{L^{\odot}}}
$$

where the current $\mathrm{I}$ is in $\mathrm{kA}$ and the gas velocity $\mathrm{V}_{\text {gas }}$ is in $\mathrm{km} / \mathrm{s}$. The other quantities entering this equation are :

$$
\begin{aligned}
\rho_{\text {gas }}= & \text { the relative density of ambient gas being snow- } \\
& \text { plow accelerated to velocity } \mathrm{V}_{\text {gas }}, \text { with unit } \\
& \text { relative density corresponding to air at STP. } \\
\mathrm{A}= & \text { the cross-sectional area, in } \mathrm{cm}^{2}, \text { of the railgun } \\
& \text { structure used to generate Lorentz force } \\
\mathrm{L}^{\prime}= & \text { the inductance per unit length, in } \mu \mathrm{H} / \mathrm{m}, \text { of the } \\
& \text { railgun structure }
\end{aligned}
$$

For example, using argon gas at $\operatorname{STP}(\rho=1.389$ times air) in a $1.6 \mathrm{~cm}^{2}$ gun structure with inductive gradient $0.5 \mu \mathrm{H} / \mathrm{m}$, to achieve a velocity of $4 \mathrm{~km} / \mathrm{s}$ requires $135 \mathrm{kA}$ driving current.

The electrical pulse width required is dependent on the fraction $\mathrm{f}$ of gas velocity to which the powder particle is to be accelerated. A practical value is $50 \%(\mathrm{f}=0.5)$. The pulse length is then given by the relation

$$
\delta \mathrm{t}=\frac{4}{3} \frac{\rho_{\text {powder }}}{\rho_{\text {gas }}} \frac{D_{\text {powder }}}{V_{\text {gas }}}\left(\frac{f}{1-f}\right)
$$

where in addition to the previously defined quantities we also have

$\delta \mathrm{t}=$ the electrical pulse duration in microseconds

$\rho_{\text {powder }}=$ the powder density relative to air at STP

$\mathrm{D}_{\text {powder }}=$ the effective diameter of the powder particles in microns

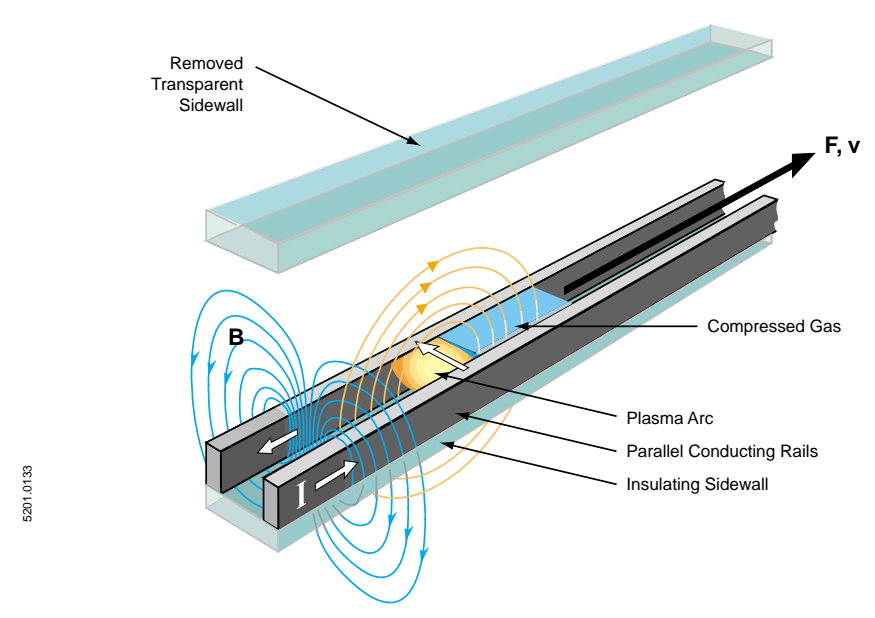

Fig. 2. The plasma armature is accelerated down the length of the railgun by an electromagnetic Lorentz force generated by the interaction of the magnetic fields surrounding the rails and the current flowing through the armature. 


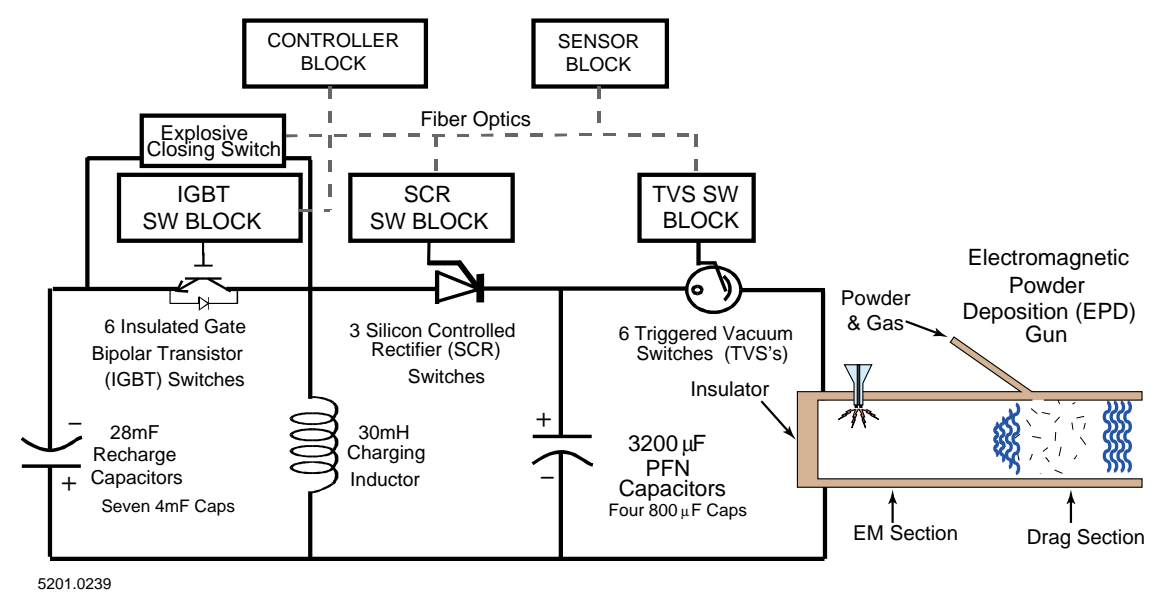

Fig. 3. The basic elements of the EPD system.

For reference, using 100 micron Inconel ${ }^{\mathrm{TM}}$ powder yields pulse lengths of about $100 \mu \mathrm{s}$.

To avoid plasma instabilities the power source must provide current to the square bore accelerator (SBA) with a rapid rise time (less than $25 \mu \mathrm{s}$ ) and the entire pulse must be very short (about $125 \mu \mathrm{s}$ ). The ideal current pulse would jump instantly to $135 \mathrm{kA}$, remain constant for about $100 \mu \mathrm{s}$ and drop instantly to $0 \mathrm{kA}$. For laboratory testing, a stage PFN has been used as a power source.

\section{Multi-Shot Power Supply}

The multi-shot power supply (MSPS) subsystem provides the pulsed power necessary to perform up to 10 repetitive discharges across the SBA. The MSPS consists of the following: a recharge capacitor bank (RCB), a charging inductor, a pulse forming network (PFN), control electronics, and power switching devices. Timed commands from the control electronics to the power switching devices transfer energy from RCB through the charging inductor to the PFN and ultimately to the SBA. A schematic for the MSPS is presented in Fig. 3. The MSPS discharge repetition rate is adjustable through the control electronics, but is initially set to be one current discharge every $30 \mathrm{~ms}$ (a rate comparable to that of the DGUN, which operates at one discharge every $125 \mathrm{~ms}$ ). Other operational parameters that may be adjusted by the MSPS control electronics are the number of discharges and the PFN current pulse magnitude. The current pulse peak magnitude is initially set to $190 \mathrm{kA}$, and its duration is approximately $100 \mu \mathrm{s}$. The total number of repetitive discharges may be extended by adding capacitance to the RCB.

In order to protect both personnel and subsystem components, the MSPS control electronics were designed with several safety features. Designated voltage and current signals are sensed throughout the MSPS functional blocks to provide feedback to the controller electronics. If a fault condition is sensed, the MSPS control electronics initiate the appropriate actions to halt system operation. The hardware that makes up the EPD system is presented in Fig. 4.

\section{EXPERIMENTAL RESULTS}

To have a reliable spray system, the results from shot to shot have to be repeatable. A Taguchi test matrix was executed to identify a set of test parameters that would optimize coating mass build-up. Typical operating parameters are $100 \mathrm{~ms}$ between shots, a PFN charge voltage of 3,400 V, and a powder mass flowrate of $22 \mathrm{~g} / \mathrm{m}$. A Nicolet Pro 40 oscilloscope was used to collect gun current and B-dot information.

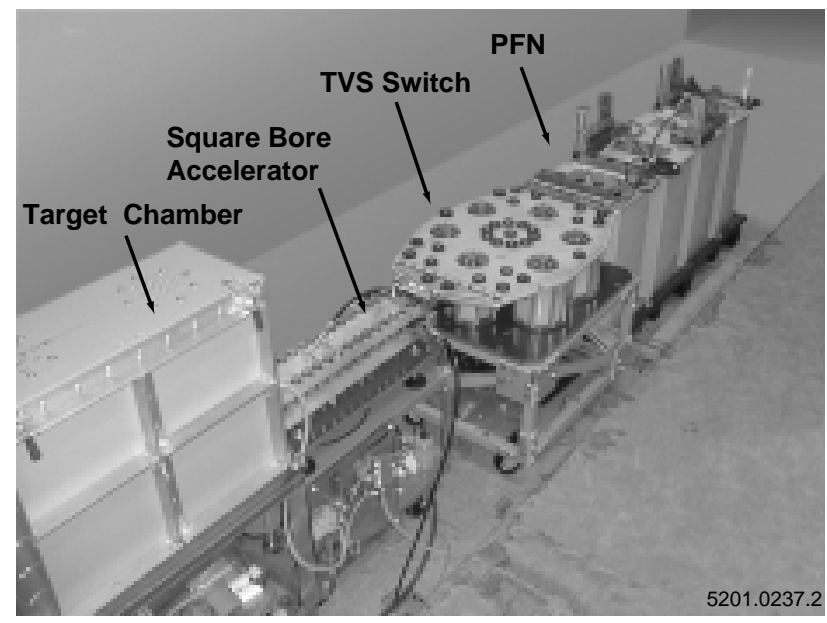

Fig. 4. Experimental hardware for pulsed power circuit. 
This scope has memory capacity for 270,000 points. If a full ten-shot burst is sampled (a $1 \mathrm{~s}$ interval), the minimum time per point is limited to $5 \mu \mathrm{s}$. This does not provide good resolution of the B-dot curves. The method of testing is to repeat two ten-shot bursts and sample the first five shots at $2 \mu$ s per point resolution and then the last five shots during the second ten shot sequence. Gun current traces from a typical ten-shot burst are presented in Fig. 5. These traces were generated from two ten-shot sequences. It can be seen that the current to the gun is very repeatable.

The spatial placement of B-dot probes down the gun are shown in Fig. 6. B-dot 1 is located $26.228 \mathrm{~cm}$ down the gun, number 2 is at $34.971 \mathrm{~cm}$ and B-dot 3 is at $43.713 \mathrm{~cm}$. Two ten-shot tests sequences, test 222 and 223 were performed to capture B-dot data. In the first test, shots 1 to 5 were sampled and in the second test, shots 6 to 10 were collected in Fig. 7. The absolute time is plotted on the time axis. From the probe data, one can construct average velocity versus time to compare with prediction. The velocity plot is presented in Fig. 8 and indicates a snowplow velocity of $4,000 \mathrm{~m} / \mathrm{s}$. The plasma reaches velocity early in the discharge, and subsequent energy is used to gather more mass into the gas column. This velocity matches simulation which models the snowplow process. If gas was not being swept up and efficiently entrained, a much higher armature velocity would be measured.

To verify that such gas flow will accelerate powder particles, insertion of 300 micron diameter, $43 \mu \mathrm{g}$ mass glass spheres into the gas flow was performed. Large particles were specifically chosen so that they would be easier to see with the magnifying lens of the camera. The glass spheres were started with zero velocity, that is they were initially at rest. The simulation code predicted that the final velocity should be $0.625 \mathrm{~km} / \mathrm{s}$. A fast CCD framing camera, rented from Hadland Corporation, was used with $1 \mu$ s interframe delay to photograph the spheres. Fig. 9 shows two consecutive frames from one shot (similar data was obtained on many shots.) The Hadland camera system indicated the spheres had a mean velocity of $0.6 \mathrm{~km} / \mathrm{s}$, in agreement with code results.

Inconel ${ }^{\mathrm{TM}}$ powder has been accelerated and used to make coatings. The mean diameter of the powder is approximately $80 \mu \mathrm{m}$. Simulations predict that the $4,200 \mathrm{~m} / \mathrm{s}$ gas column of specific duration will accelerate these particles to $2,000 \mathrm{~m} / \mathrm{s}$. A metallurgical sample of the coating has been prepared and etched to show grain structure. Fig. 10 presents a typical micrograph that demonstrates grain growth across the substrate coating interface. This is indication that a fusion bond has been achieved. In addition, the grain size of the original substrate has remained large indicating a very small heataffected zone.

\section{FUTURE DIRECTION FOR RESEARCH}

As the technology matures and future direction moves towards identifying a production system, the research effort will center around trying to identify a power supply that can drive the process on a continuous basis. The challenge will be to minimize the total energy required per pulse so that upon rep rating the power requirements remain reasonable. We have used our simulation to outline the fundamental energy requirements of the system. Quantities required of the process are: kinetic energy of the compressed gas and powder, $1.179 \mathrm{~kJ}$; arc resistive energy, $2.25 \mathrm{~kJ}$; rail resistive energy, $0.87 \mathrm{~kJ}$; for a total energy of $4.299 \mathrm{~kJ}$.

If the source is power-matched to the load, then an additional $4.299 \mathrm{~kJ}$ is absorbed by the power supply. The EPD process is rep rated at 30 shots a second to obtain the mass deposition rate required which indicates a process requiring just over $250 \mathrm{~kW}$ of prime power. This is the average power required of the supply. During the railgun pulse, a peak power

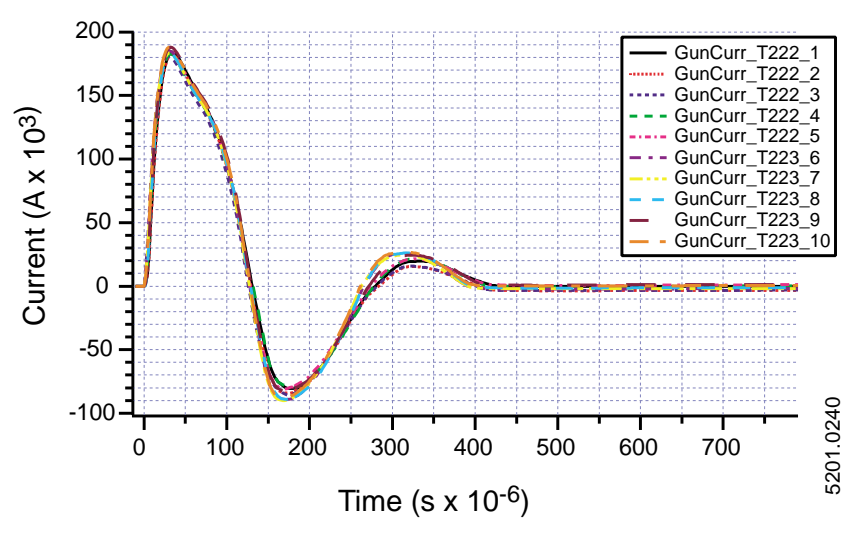

Fig. 5. Overlay of gun current from ten-shot sequence demonstrating reproducability.

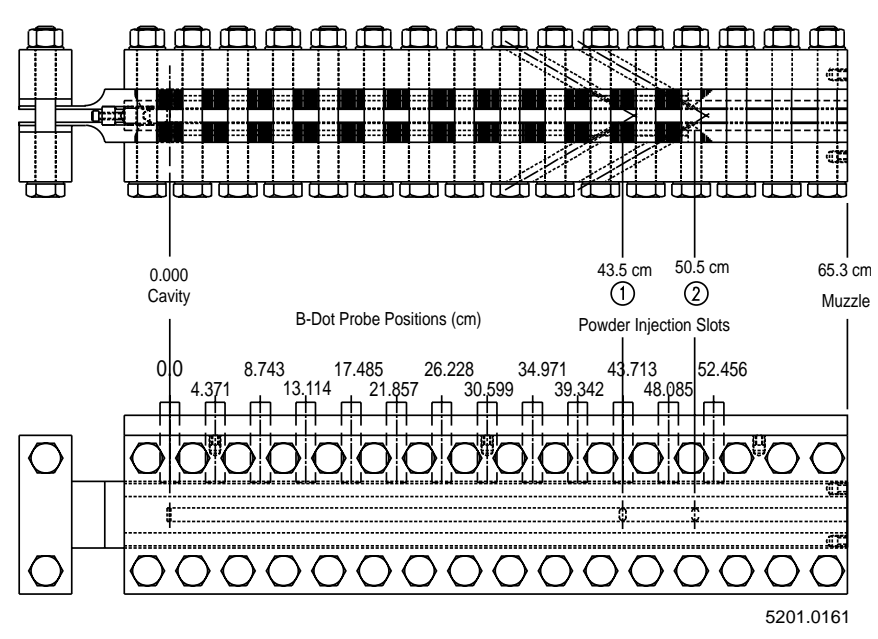

Fig. 6. SBA powder injector and B-dot positions. 

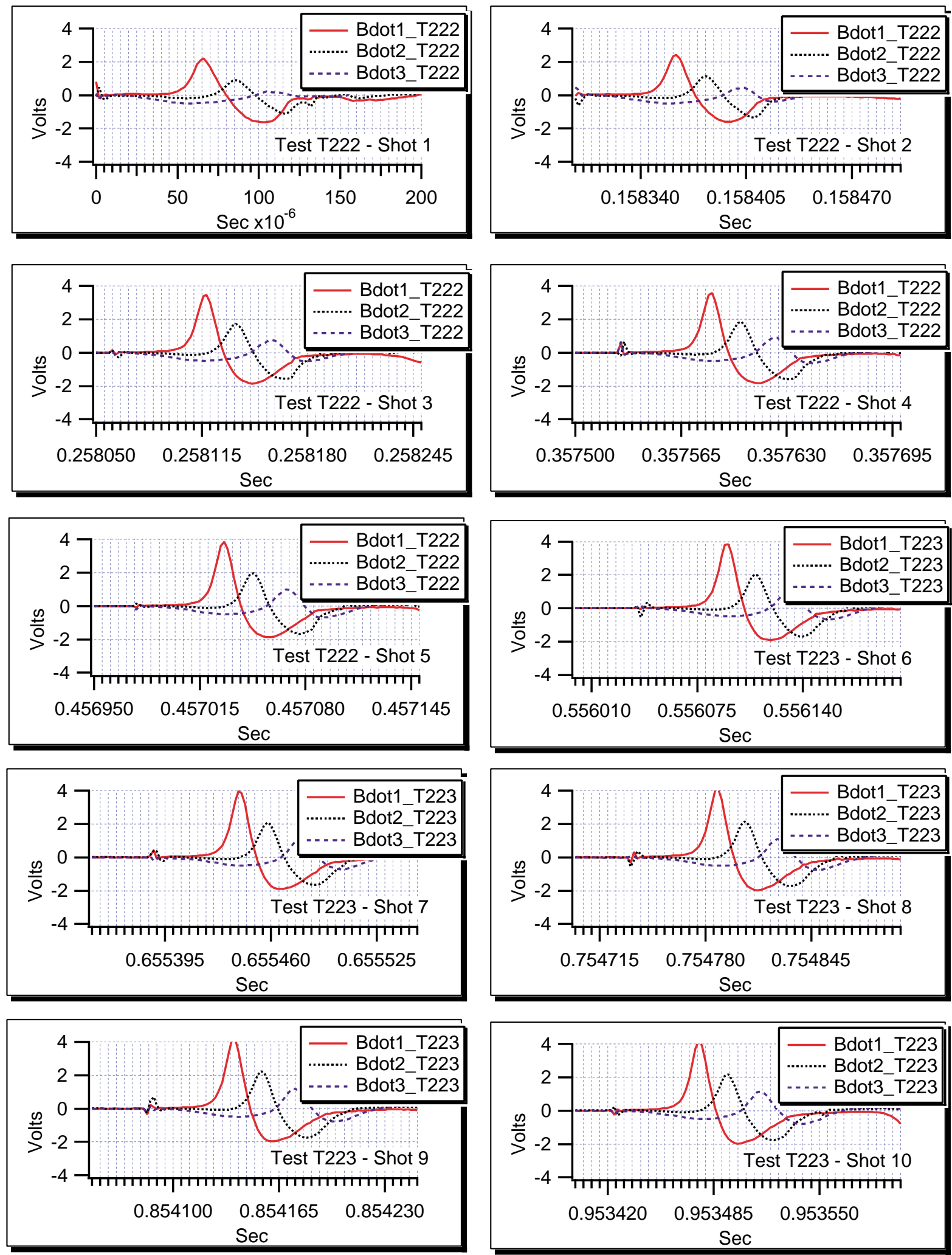

5201.0241

Fig. 7. B-dot traces from ten-shot experiment confirming simulated armature velocity and reproducability shot to shot. 


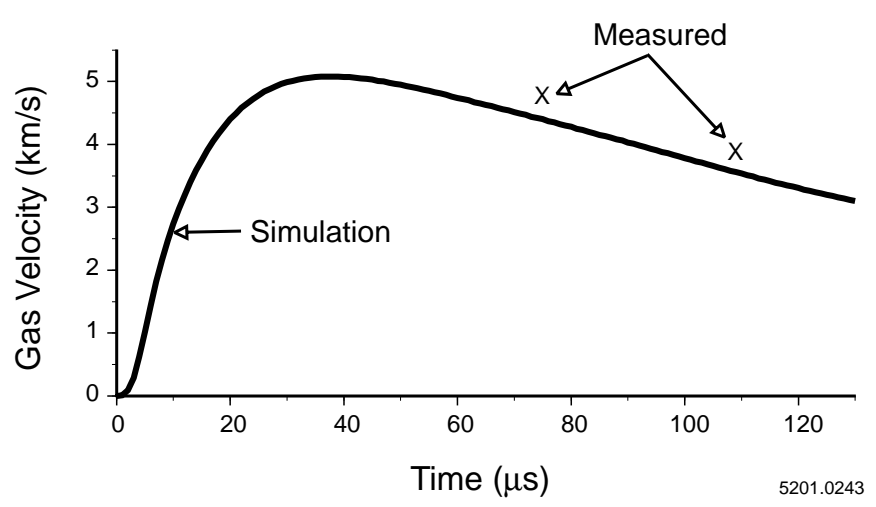

Fig. 8. Arc velocity vs. time.

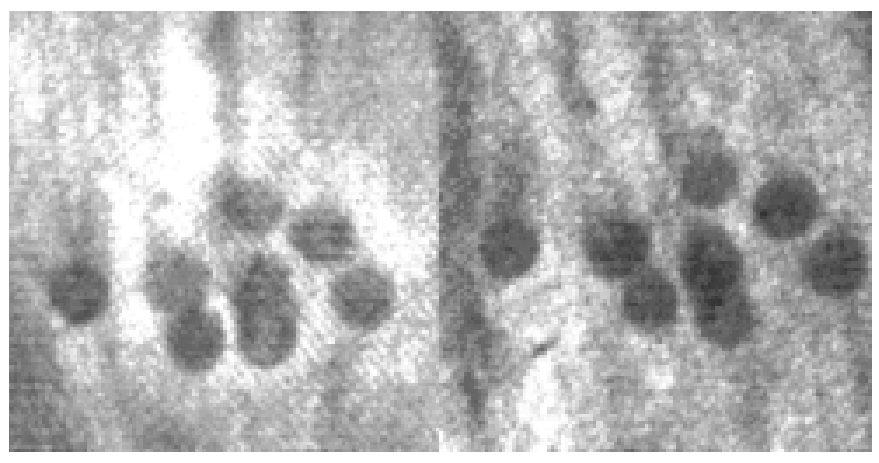

Fig. 9. Spheres at $0.6 \mathrm{~km} / \mathrm{s}$.

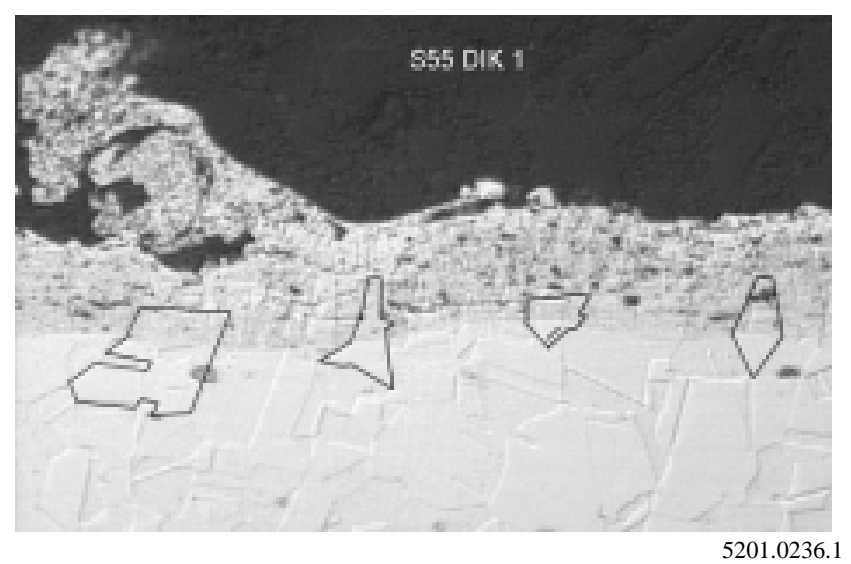

Fig. 10. Highlighted examples of grain growth across substrate coating interface.

of $80 \mathrm{MW}$ is required. The challenge is to identify a continuous rated pulsed power source capable of this power output which is properly impedance-matched to the load.

Augmented railguns are also being considered in system trade studies. As presented earlier, the peak current demand of the accelerator goes inversely with the square root of the inductance gradient. Lowering the peak current will help to control bore erosion and reduce steady state losses. It is also being determined in simulation that the higher dynamic impedance of the augmented launcher allows a more efficient match to the pulsed forming network. A bench test augmented accelerator is being designed to determine if the augmenting field will interfere with proper snowplow formation. If this testing is successful, the prime power requirement for the system will drop substantially.

Simulations assuming a snowplow process predict a velocity and duration of gas sufficient to accelerate $80 \mu \mathrm{m}$ size Inconel ${ }^{\mathrm{TM}}$ powder to $2,000 \mathrm{~m} / \mathrm{s}$. B-dot measurements of the arc that forms and accelerates the shocked gas column agree with the simulated gas velocity of $4,000 \mathrm{~m} / \mathrm{s}$. Two different camera companies will be hired to photograph the powder in flight and resolve its velocity. This independent confirmation of system performance will be combined with the metallurgical analysis of the coating bond quality, ASTM testing of coating bond strength and ductility, and ASTM testing of coating density to evaluate this ultra high velocity coating process.

\section{CONCLUSIONS}

To date, powder has been accelerated in a rep rate mode and used to form coatings on substrates. The metallurgical analysis of the coating-substrate interface indicates a fusion bond has been formed with little heat affected zone. ASTM testing of sample coupons is underway to test bond strength and coating density. The process has promise of greatly improving bond strength and coating density if empirical scaling trends continue with increasing velocity.

\section{ACKNOWLEDGMENT}

This research was sponsored by the United States Air Force, Tinker Air Force Base at Oklahoma City, through a contract administered by ARINC Corporation.

\section{REFERENCES}

[1] R. McCune, "An exploration of the cold gas-dynamic spray method for several materials systems," presented at the 8th National Thermal Spray Conference, 1995.

[2] J.W. Shearer, "Xenon shock waves driven by high magnetic fields," presented at the 2nd International Conference on Magagauss Magnetic Fields, 1979.

[3] R. Sledge, et al "Arc initiation for the electromagnetic powder deposition gun," United Thermal Spray Conference, Indianapolis, Indiana, September 1997.

[4] J. Uglum, et al, "Scaling analysis of the electromagnetic powder deposition gun," United Thermal Spray Conference, Indianapolis, Indiana, September 1997. 